\title{
Vibration analyses of composite laminates
}

\section{using a higher-order shear and normal deformable plate theory by}

\section{Chebyshev-Legendre-Galerkin method}

\begin{abstract}
Wei Wang
1 School of Urban Rail Transportation, Soochow University

2 School of Mathematical Sciences, Soochow University

China

wangw@suda.edu.cn

Sen Li

School of Mathematical Sciences, Soochow University

China

Abstract- Chebyshev Legendre Galerkin(CLG) method coupled with higher-order shear and normal deformable plate theory is proposed to analyze free and forced vibrations of laminated composite plates. The laminates of various boundary conditions, side-to-thickness ratios, and material properties are considered by present method and the numerical results agree well with their corresponding analytical solutions. High accuracy, stability and efficiency is illustrated by comparing the presented method with the other methods.
\end{abstract}

Key words - Higher order shear and normal deformable plate theory(HOSNDPT);Chebyshev-LegendreGalerkin(CLG);

Composite laiminates; Vibration

\section{INTRODUCTION}

Laminated plate and shell structure is one of the most widely used in engineering structure, so analyze the static and vibration problems of this structure is particularly important. The common analysis theory is classical Kirchhoffthin plate theory (CLT), which ignores transverse shear effects, provides reasonable results for thin plates. However, it may not obtain accurate results for moderately thick plates. A development on the CLT is the first-order shear deformation theory (FSDT) such as the Reissner Mindlin moderately thick plate theory which gives reasons for transverse shear effects, but needs a shear correction factor. Higher-order shear deformation plate theories[1,2,3,4] use higher-order polynomials to express displacement components through the plate thickness and do

\author{
Shi-Chao Yi \\ School of Mathematics and Physics ,Jiangsu University of \\ Science and Technology \\ China \\ Yishichao831029@sina.com \\ Lin-Quan Yao
}

School of Urban Rail Transportation,Soochow University

China

not require shear correction factors. Among them, the higher-order shear and normal deformable plate theory (HONSDPT)[5,6,7]takes both the transverse normal and the transverse shear deformations into account and uses Legendre polynomialsas basis functions. Prominent characteristics of the theory include the satisfaction of natural boundary conditions prescribed on both the top and the bottom surfaces of the plate, and computations of the transverse normal and the transverse shear stresses directly from the plate equations rather than by integration through the thickness of the three-dimensional balance of linear momentum. In the HOSNDPT, the three components of displacement are expanded in the thickness direction and terms up to the same degree(the polynomial basis) in the thickness coordinate are retained. In the compatible HOSNDPT, three-dimensional Hookes law is used to derive the constitutive relations for various kinetic variables in terms of the kinematic variables. The compatible HOSNDPT gives more detailed constitutive relations to bring new expressions, and the novel forms can shorten the time of calculation in exceptional circumstances. For the same order of the plate theory, the compatible HOSNDPT gives results of transverse normal and transverse shear stresses that are closer to their analytical values than the original HOSNDPT's. However, the latter is easier to implement in programming. The compatible theory has been used for analyzing static and dynamic deformations of isotropic homogeneous[5], functionally graded (FG) thick plates[8,9].

GIP of Jiangsu Province(KYLX 1214);

National Natural Science Foundation of China (No. 11172192). 
Finite element method (FEM) and boundary element method (BEM) are two common numerical methods. In FEM, construction of $\mathrm{C} 1$ conforming finite element approximation causes severe difficulties in variational formulations of thin plate model. The need of higher grid quality and density also increases the workload and complexities for problems of material inhomogeneity. As a result, the numerical solutions give the complete three dimensional displacement for the cross-section. In BEM, it is quite hard to procure the fundamental solutions for FGM problems. Spectral methods are one of the big three technologies (involved the finite difference method in 1950s, the finite element method in 1960s and the spectral method in 1970s) for the numerical solution of PDEs. For spectral methods, some of the ideas are as old as interpolation and expansion, and more specifically algorithmic developments arrived with Lanczos as early as in 1938 and with Fox, Clenshaw and others in the 1960s. If ones wants to solve an ODE or PDE to high accuracy on a simple domain and if the data defining the problem as smooth, the spectral methods are usually the best tool. They can often achieve ten digits of accuracy where a finite difference or finite element method would get three or four. They demand less computer memory than the other methods. The spectral collocation method is the most popular form of the spectral methods. It is very easy to implement, in particular for one-dimensional problems, even for very complicated nonlinear equations, and generally leads to satisfactory resultsas long as the problems process sufficient smoothness. However, the spectral collocation method needs the equilibrium equations of the problem. Spectral-Galerkin method overcomes the difficulty by involving the weak form of the equations and retains its high accuracy and high stability. The main advantage of the Chebyshev-Galerkin method is that the discrete Chebyshev transforms can be accelerated by using FFT ( Fast Fourier Transformation ) in $0\left(n \times \log _{2} n\right)$ operations. However, the Chebyshev-Galerkin method leads to non-symmetric and full stiffness matrices. On the other hand, the Legendre-Galerkin method leads to symmetric sparse matrices, but the discrete Legendre transforms are expensive $\left(O\left(n^{2}\right)\right.$ operations). In order to take advantages and overcome disadvantages of both the Chebyshev and Legendre polynomials, we use the Chebyshev-Legendre Galerkin method. For the high dimensional problems, the tensor product method is one of the most useful schemes for various PDEs. Carrera, Demasi and Fazzolari[10] analysis static and dynamic analysis of multilayered plates using the spectral methods with different plate theories in detail. High accuracy and spectral convergence are obtained by the spectral collocation method coupled with the tensor prod-uct method. Unforately, there is no mention on the higher order shear and normal deformable plate theory and the Chebyshev-Legendre Galerkin method in [10].

The remainder of the paper is organized as follows. In Section II , Spectral-Galerkin methods is described. SectionIII, the compatible higher-order shear and normal deformable plate theory is introduced in detail. In SectionIV, a Galerkin weak form are studied for the laminated plates mechanics problems. In Section $\mathrm{V}$, several examples are presented to show the computed natural frequencies of a simply supported square plate are found to match well with the corresponding analytical values. Finally, we end this paper with some conclusions in SectionVI.

\section{SPECTRAL-GALERKIN METHODS}

\section{A. Quadrature formulas}

We want to create quadrature formulas of the type

$$
\int_{a}^{b} f(x) \omega x d x \approx \sum_{i=0}^{n} A_{n} f p(
$$

If the choice of nodes $x_{0}, x_{1}, \cdots, x_{i}, \cdots x_{n}$ is made a priori, then in general the above formula is exact for polynomials of degree $\leq \mathrm{n}$. However, if we are free to choose the nodes $x_{i}$, we can expect quadrature formulas of the above form be exact for polynomials of degree up to $2 n+1$.

There are three commonly used quadrature formulas [11]. Each of them is associated with a set of collocation points which are zeros of a certain orthogonal polynomial. Table1 is the three commonly used quadrature formulas, their Chebyshev polynomial and Legendre polynomial corresponding $\left(x_{i}, \omega_{i}\right)$ in [11]. 
Table1 quadrature formulas and $\left(x_{i}, \omega_{i}\right)$

\begin{tabular}{|c|c|c|c|}
\hline & Gauss quadrature & $\begin{array}{l}\text { Gauss-radau } \\
\text { quadrature }\end{array}$ & $\begin{array}{c}\text { Gauss- lobatto } \\
\text { quadrature }\end{array}$ \\
\hline $\begin{array}{l}\text { Quadrature } \\
\text { formulas }\end{array}$ & $\begin{array}{l}\sum_{i=0}^{n} \omega_{i} p\left(x_{i}\right) \\
=\int_{a}^{b} p(x) \omega(x) d x \\
\forall p \in P_{2 n+1}\end{array}$ & $\begin{array}{l}\sum_{i=0}^{n} \omega_{i} p\left(x_{i}\right) \\
=\int_{a}^{b} p(x) \omega(x) d x \\
\forall p \in P_{2 n}\end{array}$ & $\begin{array}{l}\sum_{i=0}^{n} \omega_{i} p\left(x_{i}\right) \\
=\int_{a}^{b} p(x) \omega(x) d x, \\
\forall p \in P_{2 n-1}\end{array}$ \\
\hline $\begin{array}{c}\text { Chebyshev-po } \\
\text { lynomial } \\
\left(x_{i}, \omega_{i}\right)\end{array}$ & $\begin{array}{l}x_{i}=\cos \frac{(2 i+1) \pi}{2 n+2} \\
\omega_{i}=\frac{\pi}{n+1} \\
0 \leq i \leq n\end{array}$ & $\begin{array}{l}x_{0}=1, \\
\omega_{0}=\frac{\pi}{2 n+1}, \\
x_{i}=\cos \frac{2 i \pi}{2 n+1} \\
\omega_{i}=\frac{2 \pi}{2 n+1} \\
0 \leq i \leq n\end{array}$ & $\begin{array}{l}x_{0}=1, x_{n}=-1, \\
\omega_{0}=\omega_{n}=\frac{\pi}{2 n}, \\
x_{i}=\cos \frac{i \pi}{n}, \\
\omega_{i}=\frac{\pi}{n}, \\
1 \leq i \leq n-1\end{array}$ \\
\hline $\begin{array}{l}\text { Legendre-poly } \\
\text { nomial } \\
\qquad\left(x_{i}, \omega_{i}\right)\end{array}$ & $\begin{array}{l}x_{i} \text { are the zeros } \\
\quad \text { of } \\
L_{n+1}(x) \\
\omega_{i}=\frac{2}{\left(1-x_{i}^{2}\right)\left[L_{n+1}^{\prime}\left(x_{i}\right)\right]^{2}} \\
0 \leq i \leq n\end{array}$ & $\begin{array}{l}x_{i} \text { are the zeros of } \\
L_{n+1}(x)+L_{n}(x) \\
\omega_{0}=\frac{2}{(n+1)^{2}} \\
\omega_{i}=\frac{2}{(n+1)^{2}\left[L_{n+1}^{\prime}\left(x_{i}\right)\right]^{2}} \\
1 \leq i \leq n\end{array}$ & $\begin{array}{l}x_{i} \text { are the zeros } \\
\quad \text { of } L_{n}^{\prime}(x) \\
x_{0}=-1, x_{n}=1, \\
\omega_{i}=\frac{2}{n(n+1)\left[L_{n+1}^{\prime}\left(x_{i}\right)\right]^{2}} \\
0 \leq i \leq n\end{array}$ \\
\hline
\end{tabular}

\section{B. Spectral-Galerkin methods}

If the basis function $p_{k}(x)$ are polynomials, the spectral approximation is of the form $u^{n}(x)=\sum_{k=0}^{n} a_{k} p_{k}(x)$, where the coefficients $a_{k}$ can be determined form a given set of collocation points $x_{j}$, and the function values $u^{n}\left(x_{j}\right)$. Since $u^{n}(x)$ is a polynomial, it can be written in the form

$$
u^{n}(x)=\sum_{k=0}^{n} u^{n} \nLeftarrow F_{k} x(
$$

Where $F_{k}(x)$ are called Lagrange polynomials which satify the Kronecker delta property $F_{k}\left(x_{j}\right)=\delta_{k j}$.

If the Chebyshev polynoials are the basis functions, the spectral Galerkin method is also called Chebyshev Galerkin method. Different spectral methods have their advantages and disadvantages. We compare the Chebyshev Galerkin method(CG) with the Legendre Galerkin(LG) method.

\begin{tabular}{lll}
\hline & operations & Matrix \\
\hline CG & $0\left(n \times \log _{2} n\right)$ & Non-symmetric,full,stiffness \\
\hline LG & $0\left(n^{2}\right)$ & Symmetric,sparse \\
\hline
\end{tabular}

Then, we consider the Chebyshev-Legendre Galerkin method. Each Chebyshev-Legendre process can be seen as two steps:

(a).The process between the coefficients of the ChebyshevGauss-Lobatto nodes and the coefficients of its Cheby-shev expansion. This can be done in $0\left(n \times \log _{2} n\right)$ operations by using FFT.

(b). The relationship between the coefficients of the Chebyshev expansion and that of the Legendre expansion. Alpert and Rohklin have developed an $0(n)$ for this transform.

Therefore, the total computational cost for the ChebyshevLegendre process is of order $0\left(n \times \log _{2} n\right)$. Hence, it is most attractive for very $\operatorname{larg} n$.

Compatible higher-order shear and normal deformable plate theory Legendre polynomials Legendre polynomials $P_{0}(z), P_{1}(z), \cdots, P_{n}(z), \cdots$ are calculated for the basis $\left\{1, z, \cdots, z^{n}, \cdots\right\}$ on the interval $[-1,1]$ and the weight function is $\rho(z) \equiv 1$, the orthogonal property satisfying

$$
\int_{-1}^{1} P_{n}(z) P_{m}(z) d z=\left\{\begin{array}{cc}
0, & m \neq n \\
\frac{2}{2 n+1}, & m=n
\end{array}\right.
$$

Orthonormal Legendre polynomials calculated by the standardized for Legendre polynomials in the interval $[-h / 2, h / 2]$ and expressions for the first seven orthonormal Legendre polynomials are

$$
\begin{aligned}
& \left.L_{0}(z)=\frac{1}{\sqrt{h}}, \quad L_{\mathrm{H}} \notin\right)=2 \sqrt{\frac{3}{h}} \frac{z}{h}, \quad L_{2}(z)=\frac{1}{2} \sqrt{\frac{5}{h}}\left\{12\left(\frac{z}{h}\right)^{2}-1\right\}, \\
& L_{3}(z)=\sqrt{\frac{7}{h}}\left\{20\left(\frac{z}{h}\right)^{3}-3\left(\frac{z}{h}\right)\right\}, \quad L_{4}(z)=\sqrt{\frac{9}{h}}\left\{70\left(\frac{z}{h}\right)^{4}-15\left(\frac{z}{h}\right)^{2}+\frac{3}{8}\right\}, \\
& L_{5}(z)=\sqrt{\frac{11}{h}}\left\{252\left(\frac{z}{h}\right)^{5}-70\left(\frac{z}{h}\right)^{3}+\frac{15}{4} \frac{z}{h}\right\}, \\
& L_{6}(z)=\sqrt{\frac{13}{h}}\left\{924\left(\frac{z}{h}\right)^{6}-315\left(\frac{z}{h}\right)^{4}+\frac{105}{4}\left(\frac{z}{h}\right)^{2}-\frac{5}{16}\right\}, \\
& L_{7}(z)=\sqrt{\frac{15}{h}}\left\{3432\left(\frac{z}{h}\right)^{7}-1386\left(\frac{z}{h}\right)^{5}+\frac{315}{2}\left(\frac{z}{h}\right)^{3}-\frac{35}{8} \frac{z}{h}\right\},
\end{aligned}
$$

The standardized legendre polynomials satisfy the orthogonal conditions

$$
\int_{-h / 2}^{h / 2} L_{i}(z) L_{j}(z) d z \delta_{i j} \quad, \quad \dot{\xi} j \quad 0 ; \cdot \cdot
$$

Where $\delta_{i j}$ is the Kronecker delta function.

The derivative of the $i$-th Legendre polynomial is a polynomial of degree $i-1$, which can be linear represented by 
the first $i-1$ order Legendre polynomials. Then, it can be represented as

$$
L_{i}^{\prime}(z)=\sum_{j=0}^{K} d_{j} L(
$$

Where $d_{i j}$ is constant. For $K=7$,

$$
\left[d_{i j}\right]=\frac{2}{h}\left[\begin{array}{cccccccc}
0 & 0 & 0 & 0 & 0 & 0 & 0 & 0 \\
\sqrt{3} & 0 & 0 & 0 & 0 & 0 & 0 & 0 \\
0 & \sqrt{15} & 0 & 0 & 0 & 0 & 0 & 0 \\
\sqrt{7} & 0 & \sqrt{35} & 0 & 0 & 0 & 0 & 0 \\
0 & 3 \sqrt{3} & 0 & 3 \sqrt{7} & 0 & 0 & 0 & 0 \\
\sqrt{11} & 0 & \sqrt{55} & 0 & 3 \sqrt{11} & 0 & 0 & 0 \\
0 & \sqrt{39} & 0 & \sqrt{91} & 0 & \sqrt{143} & 0 & 0 \\
\sqrt{15} & 0 & 5 \sqrt{3} & 0 & 3 \sqrt{15} & 0 & \sqrt{195} & 0
\end{array}\right]
$$

\section{Compatible Higher-order shear and normal deformable} plate theory

For a composite laminate under transverse load, establish the corresponding three-dimensional coordinate system $o-x y z$, the region $\Omega$ is defined

$$
\Omega=\{0 \leq x \leq a, 0 \leq y \leq b, \text { and }-h / 2 \leq z \leq h / 2\}
$$

A 3D displacement function on the surface and thickness direction can be separated, and the thickness direction can be expanded by orthogonal Legendre polynomial, the displacement can be expressed as: The displacement field is assumed to be of the form

$$
\mathbf{u}(x, y, z, t)=\left\{\begin{array}{l}
u(x, y, z, t) \\
v(x, y, z, t) \\
w(x, y, z, t)
\end{array}\right\}=\sum_{i=0}^{K}\left\{\begin{array}{l}
u_{i}(x, y, t) \\
v_{i}(x, y, t) \\
w_{i}(x, y, t)
\end{array}\right\} L_{i}(z)
$$

When $K>1$, the plate theory is called higher-order theory.

The strain-displacement relationships are given as

$$
\boldsymbol{\varepsilon}=\left\{\begin{array}{c}
\varepsilon_{x} \\
\varepsilon_{y} \\
\varepsilon_{z} \\
\varepsilon_{y z} \\
\varepsilon_{z x} \\
\varepsilon_{x y}
\end{array}\right\}=\sum_{i=0}^{K}\left\{\begin{array}{c}
\frac{\partial u_{i}(x, y)}{\partial x} \\
\frac{\partial v_{i}(x, y)}{\partial y} \\
\sum_{j=0}^{K} w_{j}(x, y) d_{j i} \\
\frac{\partial w_{i}(x, y)}{\partial y}+\sum_{j=0}^{K} v_{j}(x, y) d_{j i} \\
\frac{\partial w_{i}(x, y)}{\partial x}+\sum_{j=0}^{K} u_{j}(x, y) d_{j i} \\
\frac{\partial v_{i}(x, y)}{\partial x}+\frac{\partial u_{i}(x, y)}{\partial y}
\end{array}\right\} L_{i}(z)=\sum_{i=0}^{K}\left\{\boldsymbol{\eta}_{i}\right\} L_{i}(z)
$$

Where $\boldsymbol{\eta}_{i}$ is a $6(K+1) n$ vector, its components are defined according to the formula in [8].

\section{Constitutive relations for a laminates}

For an orthotropic material such as an unidirectional composite lamina, the constitutive relations $\mathrm{C}$ in the material coordinate system is

$$
\boldsymbol{\sigma}=\left\{\begin{array}{l}
\sigma_{x} \\
\sigma_{y} \\
\sigma_{z} \\
\tau_{y z} \\
\tau_{z x} \\
\tau_{x y}
\end{array}\right\}=\left[\begin{array}{cccccc}
c_{11} & c_{12} & c_{13} & 0 & 0 & 0 \\
c_{12} & c_{22} & c_{23} & 0 & 0 & 0 \\
c_{13} & c_{23} & c_{33} & 0 & 0 & 0 \\
0 & 0 & 0 & c_{44} & c_{45} & 0 \\
0 & 0 & 0 & c_{45} & c_{55} & 0 \\
0 & 0 & 0 & 0 & 0 & c_{66}
\end{array}\right]\left\{\begin{array}{c}
\varepsilon_{x} \\
\varepsilon_{y} \\
\varepsilon_{z} \\
\gamma_{y z} \\
\gamma_{z x} \\
\gamma_{x y}
\end{array}\right\}=\mathbf{C}\left\{\begin{array}{c}
\varepsilon_{x} \\
\varepsilon_{y} \\
\varepsilon_{z} \\
\gamma_{y z} \\
\gamma_{z x} \\
\gamma_{x y}
\end{array}\right\}
$$

Where

$$
\begin{aligned}
& c_{11}=\frac{1-v_{23} v_{32}}{E_{2} E_{3} \Delta}, c_{22}=\frac{1-v_{13} v_{31}}{E_{1} E_{3} \Delta}, c_{33}=\frac{1-v_{12} v_{21}}{E_{1} E_{2} \Delta}, c_{12}=\frac{v_{21}+v_{31} v_{23}}{E_{2} E_{3} \Delta}, \\
& c_{13}=\frac{v_{31}+v_{21} v_{32}}{E_{2} E_{3} \Delta}, c_{23}=\frac{v_{32}+v_{12} v_{31}}{E_{1} E_{3} \Delta}, c_{44}=G_{23}, c_{55}=G_{31}, \\
& c_{66}=G_{12}, \Delta=\frac{1-v_{12} v_{21}-v_{23} v_{32}-v_{31} v_{13}-2 v_{21} v_{32} v_{13}}{E_{1} E_{2} E_{3}}
\end{aligned}
$$

The constitutive relations $\overline{\mathbf{C}}$ in the global coordinate system for monodinic materials can be written as $\overline{\mathbf{C}}=\mathbf{T C} \mathbf{T}^{\prime}$, and transformation $\mathbf{T}$ is

$$
\mathbf{T}=\left[\begin{array}{cccccc}
\cos ^{2} \theta & \sin ^{2} \theta & 0 & 0 & 0 & -\sin 2 \theta \\
\sin ^{2} \theta & \cos ^{2} \theta & 0 & 0 & 0 & \sin 2 \theta \\
0 & 0 & 1 & 0 & 0 & 0 \\
0 & 0 & 0 & \cos \theta & \sin \theta & 0 \\
0 & 0 & 0 & -\sin \theta & \cos \theta & 0 \\
\frac{1}{2} \sin 2 \theta & -\frac{1}{2} \sin 2 \theta & 0 & 0 & 0 & \cos 2 \theta
\end{array}\right]
$$

in which $\theta$ is the angle between the global coordinate system and the material system of each lamina.

\section{DISCRETE SYSTEM EQUATIONS AND NUMERICAL IMPLEMENTATION}

Based on the principle of minimum potential energy and the HOSNDPT, we can obtain the Galerkin weak form

$$
\begin{aligned}
& \int_{\Omega} \delta \boldsymbol{\varepsilon}^{T} \boldsymbol{\sigma} d \Omega+\int_{\Omega} \delta \mathbf{u}^{T} \mathbf{b} d \Omega+\int_{\Gamma_{t}} \delta \mathbf{u}^{T} \overline{\mathbf{t}} d \Gamma_{t} \\
& +\int_{\Omega} \delta \mathbf{u}^{T} \varsigma \dot{\mathbf{u}} d \Omega+\int_{\Omega} \delta \mathbf{u}^{T} \rho \ddot{\mathbf{u}} d \Omega=\mathbf{0}
\end{aligned}
$$

equation (2) is used to approximate the displacements in the Galerkin procedure. Then, we can obtain 


$$
\mathbf{u}=\left\{\begin{array}{l}
u \\
v \\
w
\end{array}\right\}=\sum_{i=1}^{n}\left[\begin{array}{ccc}
\varphi_{i} & 0 & 0 \\
0 & \varphi_{i} & 0 \\
0 & 0 & \varphi_{i}
\end{array}\right]\left\{\begin{array}{l}
u_{i} \\
v_{i} \\
w_{i}
\end{array}\right\}=\sum_{i=1}^{n} \boldsymbol{\Phi}_{i} \mathbf{U}_{i}
$$

Substituting Eq.(9) and Eq.(13) into Eq.(12) leads to the following total potential energy in matrix form

$$
\delta \mathbf{U}_{i}^{T}\left[\mathbf{K} \mathbf{U}_{i}+\mathbf{C} \dot{\mathbf{U}}_{\mathbf{i}}+\mathbf{M} \ddot{\mathbf{U}}_{\mathbf{i}}-\mathbf{F}\right]=\mathbf{0}
$$

Because of the arbitrariness of $\delta \mathbf{U}_{i}^{T}$ and formula (14), we can obtain the system of algebraic equations with damping:

$$
\mathbf{K U}_{\mathrm{i}}+\mathbf{C \mathbf { U } _ { \mathrm { i } }}+\mathbf{M \ddot { U } _ { \mathrm { i } }}-\mathbf{F}=\mathbf{0}
$$

in which the stiffness matrix $\mathbf{K}, \mathbf{M}$ are built from $6 \times 6$ matrices $\mathbf{K}_{i j}, \mathbf{M}_{i j}$, and the vectors $\mathrm{F}$ is built from the $6 \times 1$ vectors $\mathbf{F}_{i}$. These matrices and vectors are defined by

$$
\begin{aligned}
& \mathbf{K}_{\mathrm{ij}}=\int_{\Omega} \mathbf{B}_{\mathbf{i}}^{\mathbf{T}}\left[\mathbf{D}_{\mathrm{ij}}\right] \mathbf{B}_{\mathbf{j}} d \Omega ; \quad \mathbf{M}_{\mathbf{i j}}=\int_{\Omega} \mathbf{\Phi}_{\mathbf{i}}^{\mathbf{T}}\left[\rho_{i j}\right] \mathbf{\Phi}_{\mathbf{j}} d \Omega ; \\
& \mathbf{F}_{i}=\int_{\Omega} \boldsymbol{\Phi}_{\mathbf{i}} \mathbf{b} d \Omega+\int_{\Gamma_{t}} \boldsymbol{\Phi}_{\mathbf{i}} \overline{\mathbf{t}} d \Gamma, i=1,2, \cdots, k \\
& {\left[\mathbf{D}_{i j}\right]=\int_{-h / 2}^{h / 2} L_{i}(z) \mathbf{D} L_{j}(z) d z ; \quad\left[\rho_{i j}\right]=\int_{-h / 2}^{h / 2} L_{i}(z) \rho L_{j}(z) d z ;}
\end{aligned}
$$

Where $B_{i}$ is $6(K+1) \times 3(K+1)$ matrice, Its $\mathrm{i}$-th 6 forms are as follows

$\left[\begin{array}{cccccccccc}\overbrace{0}^{0} & 0 & 0 & & \overbrace{\varphi_{j, x}}^{0} & 0 & & 0 & 0 & 0 \\ 0 & 0 & 0 & 0 & \varphi_{j, y} & 0 & 0 & 0 & 0 \\ 0 & 0 & \varphi_{j} d_{0 i} & 0 & 0 & \varphi_{j} d_{i i} & 0 & 0 & \varphi_{j} d_{K i} \\ 0 & \varphi_{j} d_{0 i} & 0 & 0 & \varphi_{j} d_{i i} & \varphi_{j, y} & 0 & \varphi_{j} d_{K i} & 0 \\ \varphi_{j} d_{0 i} & 0 & 0 & \varphi_{j} d_{i i} & 0 & \varphi_{j, x} & \varphi_{j} d_{K i} & 0 & 0 \\ 0 & 0 & 0 & \varphi_{j, y} & \varphi_{j, x} & 0 & 0 & 0 & 0\end{array}\right]$

The Rayleigh damping matrix $\mathbf{C}$ is defined as a liner combination of the $\mathbf{K}$ and $\mathbf{C}, \mathbf{C}=\alpha_{1} \mathbf{M}+\alpha_{2} \mathbf{K}, \alpha_{1}, \alpha_{2}$ are constants independent to the frequency.

A general solution of the free vibration equation can be written as

$$
\mathbf{U}=\mathbf{W} \cdot e^{i \omega t}
$$

where $i$ is the imaginary unit, $t$ is the time, $\bar{w}$ is the eigenvector and $\omega$ is the natural frequency. Substituting Eq. (16) into Eq.(15) with $\mathbf{C = 0}$ and $\mathbf{F}=\mathbf{0}$, the natural frequency $\omega$ of the plate vibration can be found by solving the following eigenvalue equation

$$
\left(\mathbf{K}-\omega^{2} \mathbf{M}\right) \mathbf{W}=
$$

We use a background cell of 16 Gaussian points for the purpose of numerical integration to compute the stiffness and mass matrices. Furthermore, the boundary condition can be easily imposed as in the conventional FEM because of possessing the Kronecker delta property of the interpolation functions.

The Newmark family of methods is used to numerically discrete the system of coupled second-order PDEs on time. The recursive relation among displacements $\mathbf{U}$, velocities $\dot{\mathbf{U}}$ and acceleration $\ddot{\mathbf{U}}$ at time tn and $t_{n+1}=t_{n}+\delta_{t}$ are

$$
\begin{gathered}
\mathbf{U}^{n+1}=\mathbf{U}^{n}+\delta_{t} \dot{\mathbf{U}}^{n}+\frac{\left(\delta_{t}\right)^{2}}{2}\left[(1-2 \beta) \ddot{\mathbf{U}}^{n}+2 \beta \ddot{\mathbf{U}}^{n+1}\right], \\
\dot{\mathbf{U}}^{n+1}=\dot{\mathbf{U}}^{n}+\delta_{t}\left[(1-\gamma) \dot{\mathbf{U}}^{n}+\gamma \dot{\mathbf{U}}^{n+1}\right]
\end{gathered}
$$

$\beta, \gamma$ are constants. We set $\beta=0.25, \gamma=0.5$, then the Newmark method is equivalent to the constant average acceleration method and unconditionally stable .

Rewriting Eq.(15) at time $t n+1$, and substituting from Eq. $(18,19)$ give the following system of algebraic equations

$$
\mathbf{K}^{n+1} \mathbf{U}^{n+1}=\mathbf{F}^{n+}
$$

Where

$$
\begin{gathered}
\mathbf{K}^{n+1}=\mathbf{K}^{n}+\frac{4}{(\delta t)^{2}} \mathbf{M}^{n+1}+\frac{4}{\delta t} \mathbf{C}^{n}, \\
\mathbf{F}^{n+1}=\mathbf{F}^{n}+\mathbf{M}^{n+1}\left(\frac{4}{(\delta t)^{2}} \mathbf{U}^{n}+\frac{4}{\delta t} \dot{\mathbf{U}}^{n}+\ddot{\mathbf{U}}^{n}\right)+\mathbf{C}^{n}\left(2 \mathbf{U}^{n}+\dot{\mathbf{U}}^{n}\right)
\end{gathered}
$$

Having computed $\mathbf{U}^{n+1}$ from Eq.(20), $\ddot{\mathbf{U}}^{n+1}$ and $\dot{\mathbf{U}}^{n+1}$ are obtained from

$$
\begin{aligned}
\ddot{\mathbf{U}}^{n+1} & =\frac{4}{(\delta t)^{2}}\left(\mathbf{U}^{n+1}-\mathbf{U}^{n}\right)-\frac{4}{\delta t} \dot{\mathbf{U}}^{n}-\ddot{\mathbf{U}}^{n}, \\
\dot{\mathbf{U}}^{n+1} & =\dot{\mathbf{U}}^{n}+\frac{\delta t}{2}\left(\ddot{\mathbf{U}}^{n}+\ddot{\mathbf{U}}^{n+1}\right)
\end{aligned}
$$

\section{NUMERICAL EXAMPLES}

To verify the accuracy and convergence of the present CLG method coupled with HOSNDPT, several numerical examples are studied on free vibrations and forced vibrations problems in this section. The numerical results for these examples are compared with the analytical solutions and some reference solutions. Square influence domains are used for calculations in the present paper with the average node distance $\mathrm{dc}=3$. 


\section{A. Free vibration}

A rectangle laminated plate of length $a$, width $b$ and thickness $\mathrm{h}$ subjected to a uniformly distributed load $\mathrm{q}$ are analyzed. non-dimensionalize natural frequencies.

$$
\bar{\omega}=\frac{\omega b^{2}}{h} \sqrt{\rho / E_{2}}
$$

The relative values of material properties are as

material1: $E_{1}: E_{2}: E_{3}=25: 1: 1, v_{12}=v_{13}=v_{23}=0.25$,

$$
G_{12}=G_{13}=0.5 E_{2}, G_{23}=0.2 E_{2}, \rho=1 \mathrm{~g} / \mathrm{cm}^{3}
$$

material $2: E_{1}: E_{2}: E_{3}=40: 1: 1, v_{12}=v_{13}=v_{23}=0.25$,

$$
G_{12}=G_{13}=0.6 E_{2}, G_{23}=0.2 E_{2}, \rho=1 \mathrm{~g} / \mathrm{cm}^{3}
$$

In Table 2, the 2-layer $0^{0} / 90^{\circ}$ cantilever plate with various length-width ratio are analyzed to verify the validity of the HOSNDPT scheme coupled with the CLG method. The non-dimensionalized natural frequencies are presented in Table 2 for material1 are compared with the CLPT solutions in [12], the FSDT solutions in [12] and the TSDT solutions in [12]. It should be noted that the solutions of CLG is between these three methods and close to the TSDT solutions.

Table 2: CLG solutions coupled with other solutions of non-dimensionalized natural frequencies for a cantilever plate $\left(h / a=0.1,0^{\circ} / 90^{\circ}\right)$

\begin{tabular}{ccccccc}
\hline Method & CLPT & FSDT & TSDT & CLG(K=1) & CLG(K=3) & CLG(K=5) \\
\hline $\mathrm{b} / \mathrm{a}=1$ & 2.625 & 2.533 & 2.561 & 2.139 & 2.133 & 2.129 \\
$\mathrm{~b} / \mathrm{a}=2$ & 10.459 & 9.350 & 9.599 & 8.557 & 8.532 & 8.519 \\
$\mathrm{~b} / \mathrm{a}=3$ & 23.378 & 18.849 & 19.833 & 19.255 & 19.197 & 19.167 \\
\hline
\end{tabular}

CLG solutions of non-dimensionalized natural frequency under different lamination schemes and side-to-thickness ratio are presented in Tables 3 and 4 for material1 and material2, and are compared with the CLPT,FSDT solutions in [12].

Table 3: CLG solutions of non-dimensionalized natural frequencies under different schemes for the laminates

\begin{tabular}{ccccc}
\hline $\mathrm{h} / \mathrm{a}$ & method & $0^{0}$ & $0^{0} / 90^{\circ} / 0^{\circ}$ & $0^{\circ} / 90^{\circ} / 0^{\circ} / 90^{\circ} / 0^{\circ}$ \\
\hline & FSDT & 8.909 & 8.766 & 9.215 \\
& CLPT & 14.750 & 14.750 & 14.750 \\
0.2 & CLG $(\mathrm{K}=1)$ & 9.4059 & 9.0904 & 9.6327 \\
& CLG $(\mathrm{K}=3)$ & 8.9643 & 8.1262 & 8.9934 \\
& $\mathrm{CLG}(\mathrm{K}=5)$ & 8.9602 & 8.9934 & 8.8322 \\
\hline
\end{tabular}

\begin{tabular}{lllll}
\hline & FSDT & 12.452 & 12.227 & 12.633 \\
0.1 & CLPT & 15.104 & 15.104 & 15.104 \\
& CLG(K=1) & 12.7948 & 12.5267 & 12.8938 \\
& CLG(K=3) & 12.4631 & 11.7482 & 12.4767 \\
& CLG(K=5) & 12.4628 & 11.6180 & 12.3760 \\
\hline \multirow{2}{*}{0.02} & FSDT & 15.077 & 15.055 & 15.067 \\
& CLGPT & 15.223 & 15.223 & 15.223 \\
& CLG(K=3) & 15.1008 & 15.0805 & 15.1052 \\
& & 15.0768 & 15.0142 & 15.0762 \\
& CLG(K=5) & 15.0768 & 14.9998 & 15.0695 \\
\hline
\end{tabular}

\begin{tabular}{|c|c|c|c|c|c|c|c|}
\hline \multirow{2}{*}{$\mathrm{h} / \mathrm{a}$} & \multirow{2}{*}{ method } & \multicolumn{2}{|c|}{$\theta=5^{0}$} & \multicolumn{2}{|c|}{$\theta=30^{\circ}$} & \multicolumn{2}{|c|}{$\theta=45^{0}$} \\
\hline & & $\mathrm{Ne}=2$ & $\mathrm{Ne}=6$ & $\mathrm{Ne}=2$ & $\mathrm{Ne}=6$ & $\mathrm{Ne}=2$ & $\mathrm{Ne}=6$ \\
\hline \multirow{5}{*}{0.25} & FSDT & 8.531 & 8.737 & 8.917 & 10.502 & 9.161 & 10.805 \\
\hline & CLPT & 14.514 & 16.563 & 13.012 & 21.647 & 13.506 & 13.766 \\
\hline & $\operatorname{CLG}(\mathrm{K}=1)$ & 9.137 & 9.314 & 10.182 & 11.296 & 10.558 & 11.650 \\
\hline & $\operatorname{CLG}(\mathrm{K}=3)$ & 8.689 & 8.799 & 9.149 & 10.207 & 9.387 & 10.510 \\
\hline & CLG $(\mathrm{K}=5)$ & 8.669 & 8.781 & 9.010 & 10.143 & 9.234 & 10.446 \\
\hline \multirow{5}{*}{0.1} & FSDT & 14.179 & 14.840 & 12.681 & 18.226 & 13.044 & 19.025 \\
\hline & CLPT & 17.500 & 18.819 & 14.031 & 23.165 & 14.439 & 24.611 \\
\hline & $\operatorname{CLG}(\mathrm{K}=1)$ & 14.828 & 15.352 & 15.156 & 19.019 & 15.821 & 19.951 \\
\hline & CLG $(\mathrm{K}=3)$ & 14.397 & 14.843 & 14.194 & 17.842 & 14.657 & 18.615 \\
\hline & $\operatorname{CLG}(\mathrm{K}=5)$ & 14.387 & 14.839 & 13.916 & 17.785 & 14.323 & 18.548 \\
\hline
\end{tabular}

Table 4: CLG solutions of non-dimensionalized natural frequencies under different lamination schemes for the laminated plate $(\theta /-\theta / \ldots)$

Different boundary conditions also affect the natural frequency. Hence, the non-dimensionalized natural frequency under different boundary conditions and side-to-thickness ratio for material 2 are presented in Tables 5 and 6,and are compared with the CLPT,FSDT, TSDT solutions in [12]. are shown that the results of the present method agrees well with the reference solutions.

Table 5: CLG solutions of non-dimensionalized natural frequencies under different boundary conditions for the laminates $\left(0^{\circ} / 90^{\circ}\right)$

\begin{tabular}{cccccc}
\hline $\mathrm{h} / \mathrm{a}$ & method & SSSS & SSSC & SSSF & SCSC \\
\hline & FSDT & 10.473 & 12.610 & 7.215 & 15.152 \\
& CLPT & 11.154 & 14.223 & 7.636 & 18.543 \\
0.1 & TSDT & 10.568 & 12.870 & 7.277 & 15.709 \\
& CLG $(\mathrm{K}=1)$ & 10.578 & 12.836 & 7.279 & 15.575 \\
& CLG $(\mathrm{K}=3)$ & 10.426 & 12.495 & 7.170 & 14.938 \\
& $\mathrm{CLG}(\mathrm{K}=5)$ & 10.339 & 12.316 & 7.103 & 14.635 \\
\hline
\end{tabular}




\begin{tabular}{rccccc}
\hline & FSDT & 8.833 & 9.882 & 6.213 & 10.879 \\
& CLPT & 10.721 & 13.627 & 7.450 & 17.741 \\
0.2 & TSDT & 9.087 & 10.393 & 6.387 & 11.890 \\
& CLG(K=1) & 9.085 & 10.226 & 6.083 & 11.468 \\
& CLG(K=3) & 8.716 & 9.655 & 6.083 & 10.695 \\
& CLG(K=5) & 8.531 & 9.421 & 5.953 & 10.429 \\
\hline
\end{tabular}

Table 6: CLG solutions of non-dimensionalized natural frequencies under different

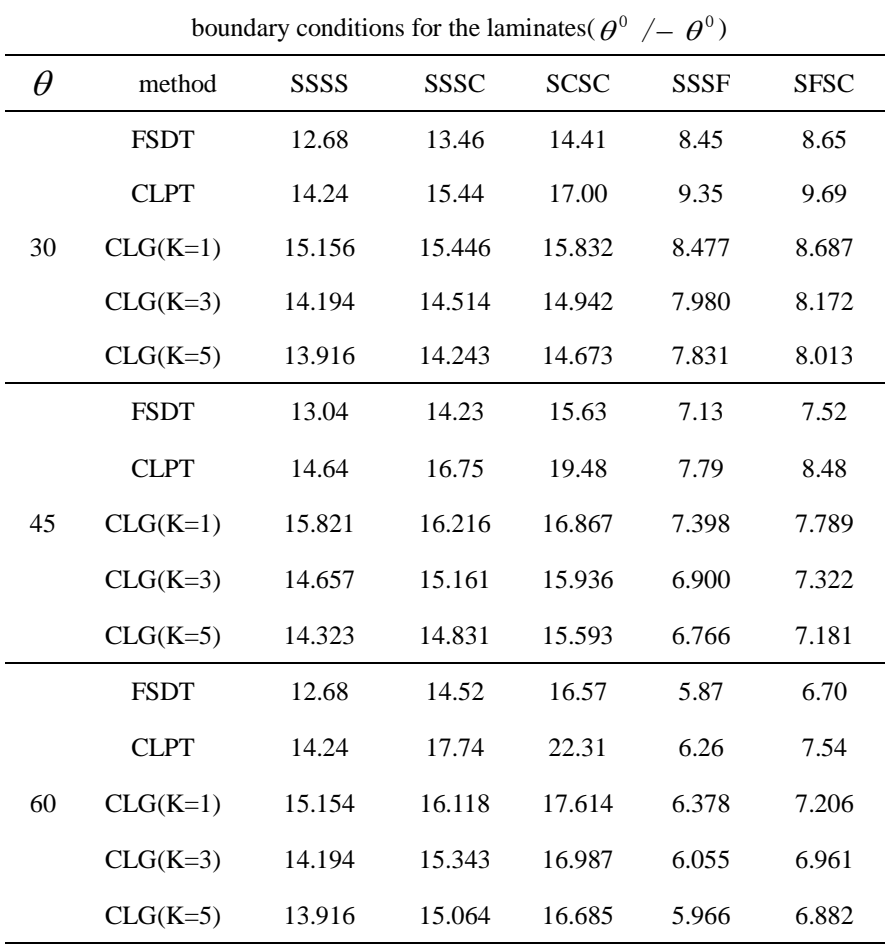

\section{B. Forced vibration}

We now consider the forced responses of the laminated plates. The relative values of material properties are as

$$
\begin{aligned}
& a=b=25 \mathrm{~cm} \text { l } 1 \subset E_{1}: E_{2}: E_{\overline{3}}=25: 1: E F, \\
& G_{12}=G_{13}=0.5 E_{2}, G_{23}=0.2 E_{2}, v_{12}=v_{23}=v_{13}=0.25, \\
& \rho=8 \times 10^{-6} \mathrm{~kg} / \mathrm{mm}^{3}, \delta_{t}=5 \mu \mathrm{s}
\end{aligned}
$$

The expression of load in this paper is $q(x, y, t)=q_{0} f(t)$, here $q_{0}=1, f(t)$ has three schemes,

- Accumulative load: $f(t)=1, t \geq 0$

- Pulse load: $f(t)=\left\{\begin{array}{cc}1 & 0 \leq t \leq 7000 \delta_{t} \\ 0 & t>7000 \delta_{t}\end{array}\right.$

- Sinusoid load: $f(t)=\left\{\begin{array}{cc}\sin \left(\pi t / t_{1}\right) & 0 \leq t \leq 7000 \delta_{t} \\ 0 & t>7000 \delta_{t}\end{array}\right.$
Fig.1 shows the non-dimensionalized center deflection versus time for cross-ply laminates $0^{\circ} / 90^{\circ}$ under uniformly distributed accumulative load without the damping effect, and is compared with the Navier solutions[11] of Reddy. Similar results are presented for $\mathrm{K}=1, \mathrm{~K}=3$ and $\mathrm{K}=5$, and the FSDT collocation method in [13] with theMQ function is compared. Results show CLG method much better than the FSDT-MQ in [13] does.

The pulse loads and sinusoid pulse loads, are considered in Fig. 2 and 3 for a 2-layer laminate $0^{\circ} / 90^{\circ}$. The parameter $t_{1}$ is taken as $7000 \delta_{t}$ Under the action of pulse load, the $\mathrm{CLG}(\mathrm{K}=1$, 3, and 5) are plotted in Fig.2. When $t \leq 7000 \mu$ s, it makes harmonic vibration near the equilibrium position $\bar{w}=1.8$ due to the external force $q_{0}$. when $\mathrm{t}>7000 \mu \mathrm{s}$, it makes harmonic vibration near the equilibrium position $\bar{w}=0$ for the deficiency of external force. Under the sinusoid pulse loads, the $\mathrm{CLG}(\mathrm{K}=1$, and 3$)$ and the absolute error between them are considered. When $\mathrm{t} \leq 7000 \mu \mathrm{s}$, it is no longer make harmonic vibration due to the influence of external force $q_{0} \sin \left(\pi t / t_{1}\right)$, when $\mathrm{t}>7000 \mu \mathrm{s}$, it is still make harmonic vibration near the equilibrium position $\bar{w}=0$ for the deficiency of external force. As can be seen in Fig.3, there is a good agreement between the different $\mathrm{K}$ of the present CLG method.

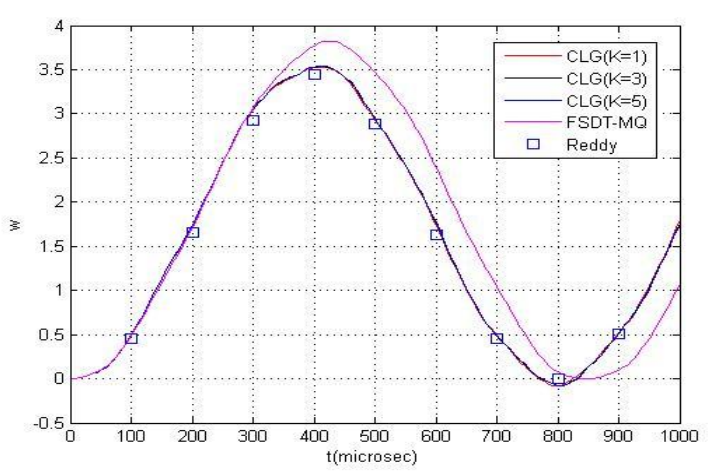

Figure 1: Center deflection versus time for different orders $\left(0^{\circ} / 90^{\circ}\right)$ under uniformly distributed accumulative load 


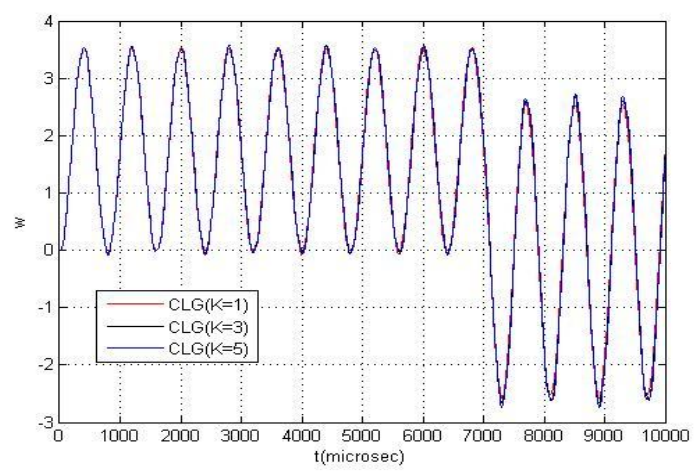

Figure 2: Vibrations curve under pulse load

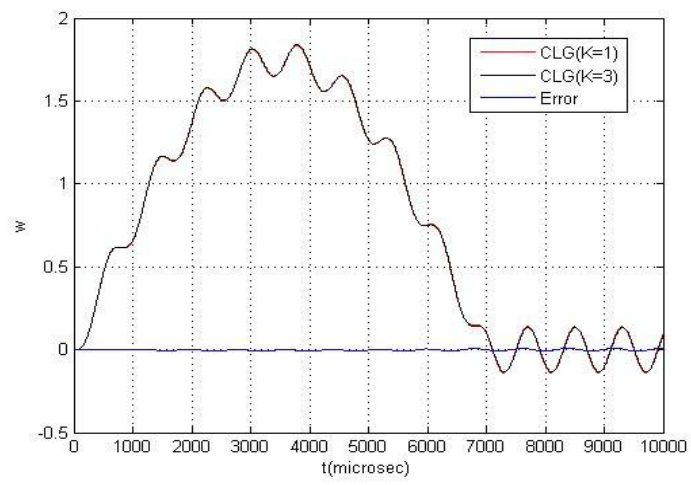

Figure 3: Vibrations curve under sinusoid pulse loads

\section{CONCLUSIONS}

CLG method with higher-order shear and normal deformation theory for the vibration of laminated plates was proposed. Several well-known plate and laminate benchmark examples were also solved. The obtained results, and the experience acquired along the development of this work, permit to conclude the following:

(a) The present method is more stable and accurate then the MLPG.

(b) In general, the convergence rate is high and the final converged solution is always very close to the considered problem analytical solution.

(c) The scheme consider all the six stresses and it is a true 3D method.

The CLG method with higher-order shear and normal deformation theory proved to be an efficient and alternative method in the dynamic analysis of composite laminates.

\section{References}

[1] Lo KH, Christensen RM, Wu EM. A higher-order theory of plate deformation. Journal of Applied Mechanics 1997;44:663-76.

[2] Phan ND, Reddy JN. Analysis of laminated composite plates using a higher-order shear deformation theory. International Journal for Numerical Methods in Engineering 1985;21: 2201-19.

[3] Batra RC, Vidoli S. Higher-order piezoelectric plate theory derived from a three-dimensional variational principle. AIAA journal 2002;40(1): 91-104.

[4] Kocak S, Hassis H. A higher order shear deformable finite element for homogeneous plates. Eng Struct 2003;25:131-9.

[5] Batra RC, Aimmanee S. Vibrations of thick isotropic plates with higher order shear and normal deformable plate theories.Computers and Structures 2005;83:934-55.

[6] Qian LF, Batra RC, Chen LM. Elastostatic deformations of a thick plate by using a higher-order shear and normal deformable plate theory and two meshless local Petrov-Galerkin (MLPG) methods. Computer Modeling in Engineering and Sciences 2003;4(1): 161-176.

[7] Xiao JR, Gilhooley DF, Batra RC, et al. Analysis of thick composite laminates using a higher-order shear and normal de-formable plate theory (HOSNDPT) and a meshless method. Composites Part B: Engineering 2008;39(2): 414-427.

[8] Qian LF, Batra RC, Chen LM. Static and dynamic deformations of thick functionally graded elastic plate by using higher-order shear and normal deformable plate theory and meshless local Petrov-Galerkin method. Composites Part B: Engineering 2004;35:685-97.

[9] Qian LF, Batra RC. Transient thermoelastic deformations of a thick functionally graded plate. Journal of Thermal Stresses 2004;27:705-40.

[10] Carrera E, Demasi L, Fazzolari F. Spectral methods for static and dynamic analysis of multilayered plates. 2010.

[11] Lloyd N.Trefethen.Spectral methods in MATLAB.SIAM press;2000.

[12] Reddy JN. Mechanics of laminated composite plates. CRC press; 1996.

[13] Chen FJ, Wei CZ, Yao LQ. Free vibration analysis of laminated composite plates by local moving Kriging meshless method. Journal of Applied Mechanics 2013, 30(4): 559-564. 